\title{
El teletrabajo desde la perspectiva de la salud laboral
}

\author{
Docente \\ Mónica María Quiroz Rubiano \\ mquiroz@poligran.edu.co \\ Estudiantes \\ Alejandra Idárraga Areiza \\ Cristian Ramírez Londoño
}

Amelia González Mesa

Institución Universitaria Politecnico Grancolombiano

Profesional en Gestión de la Seguridad y la salud laboral

Inicialmente, el la modalidad de teletrabajo, semillero de Salud Laboral, pretende ahondar en diferentes factores relacionados con promoción de la salud física, mental y prevención de la enfermedad en contextos laborales desde la perspectiva académica, a través de la identificación y valoración de los riesgos emergentes con el fin de plantear alternativas de prevención que apunten a poblaciones de alto impacto como:

1. Investigar en aspectos relacionados con la salud laboral

2. Generar propuestas de mejora en procesos encaminados a la prevención de la enfermedad.

3. Valoración de necesidades proyectadas al mejoramiento de la salud física y mental.

En esta primera fase del semillero se pretende focalizar esfuerzos en la salud ergonomíca del colaborador que realiza sus funciones en es por ello que, en medio del trabajo del grupo de estudiantes del semillero, junto con la docente líder, se ha focalizado sus esfuerzos en esta área de estudio, identificando una oportunidad de estudio con referencia a la ergonomía de los educadores del Politécnico Grancolombiano, a raíz del cambio de la dinámica del país por la enfermedad COVID-19, al identificar esta situación se pretende conocer todas aquellas situaciones que se pueden convertir en riesgos potenciales al prestar sus servicios, utilizando el apoyo tecnológico se pretende establecer, como mejorar los ambientes de trabajo, de acuerdo al contexto en el que se desempeñan en este momento.

Muy posiblemente, en las circunstancias en que se evalúen los diferentes factores de riesgos a los que están expuestos los teletrabajadores en sus ambientes de trabajo que por el momento son sus hogares, se identificarán otros factores que de igual forma puedan incidir en el bienestar de estos como son el psicosocial, factor que se puede presentar por diversas razones como el aislamiento, complejidad en la dinámica familia - trabajo, dificultad en el acceso tecnológico y complejidad en el manejo de los horarios, esto se convierte en un conjunto de situaciones de su vida diaria. Después de identificar todas estas situaciones, el proyecto semillero pretende dar una solución práctica por medio de una cartilla de autocuidado para todos aquellos docentes que se desempeñan en este momento desde esta modalidad de empleo, para aportar al mejoramiento de su salud laboral por medio de la misma.

Cabe resaltar que, actualmente por la situación mundial actual, el teletrabajo sufre una variación, del 
termino teletrabajo a aquel que cumple con ciertos criterios contractales, con suministros de herramientas $\mathrm{y}$ equipos, acceso a redes $\mathrm{y}$ telecomunicaciones, adecuación de espacios de trabajo en términos de prevención de riesgos laborales, entre otros; a una nueva modalidad de "trabajo en casa" donde en muchas ocasiones el colaborador no cuenta con ninguna de estas herramientas y el mismo debe acomodar al alcance de sus posibilidades un equipo con el cual trabajar, el acceso a redes, sin cambio en términos contractuales $y$ adaptando el ambiente del hogar como su espacio de trabajo.

Es por ello por lo que, al término de este proyecto y unificando las pretensiones del semillero junto con este proyecto se pretende cumplir el objetivo de “identificar si las practicas ergonómicas para el ejercicio del teletrabajo de los tutores virtuales de la Institución Universitaria Politécnico Grancolombiano en Bogotá Colombia, indicen en su salud por medio de molestias físicas, enfermedades o accidentes".

Esto permitirá establecer una oportunidad para generar estilos de vida saludable en los colaboradores bajo la modalidad de teletrabajo y con esto, la evolución de accidentes de trabajo durante el desempeño de esta actividad, además, enfermedades laborales que sobrevengan del riesgo ergonómico a través de la aplicación de una fase diagnóstica de los hábitos de teletrabajo, incorporando el análisis de la información recopilada y la formulación de una guía de buenas prácticas ergonómicas.

Con ello, se proyecta como resultado derivado de la investigación, generar y adoptar las medidas ergonómicas que permitan llegar al diagnóstico y surgimiento de la enfermedad asociada al teletrabajo; realizar guías de buenas prácticas que permitirán conocer las pautas para el teletrabajo y convertir el hogar de los colaboradores del Politécnico Grancolombiano, en escenarios de trabajo saludables, proporcionando consejos sobre cómo optimizar nuestros objetos caseros para mejorar ese ambiente de trabajo en casa. 